\title{
Cosmography by gamma ray bursts
}

\author{
S. Capozziello ${ }^{1}$ and L. Izzo ${ }^{1,2,3}$ \\ 1 Dipartimento di Scienze Fisiche, Università di Napoli "Federico II" and INFN Sez. di Napoli, Compl. Univ. Monte S. Angelo, \\ Ed. N, via Cinthia, 80126 Napoli, Italy \\ e-mail: capozziello@na.infn.it \\ 2 ICRANet and ICRA, Piazzale della Repubblica 10, 65122 Pescara, Italy \\ 3 Dip. di Fisica, Università di Roma "La Sapienza", Piazzale Aldo Moro 5, 00185 Roma, Italy
}

Received 6 June 2008 / Accepted 11 August 2008

\section{ABSTRACT}

\begin{abstract}
Aims. Relations connecting gamma ray burst quantities can be used to constrain cosmographic parameters of the Hubble law at medium-high redshifts.

Methods. We consider a sample of 27 gamma ray bursts to construct the luminosity distance to redshift relation and derive the values of the parameters $q_{0}, j_{0}$, and $s_{0}$. The analysis is compared with other methods in the literature.

Results. Gamma gay bursts, if calibrated by SNeIa, seem reliable as distance indicators and give cosmographic parameters in agreement with the $\Lambda$ CDM model.
\end{abstract}

Key words. gamma rays: bursts - cosmology: cosmological parameters - cosmology: distance scale

\section{Introduction}

A class of very accurate standard candles, the supernovae Ia (SNeIa), has been highly developed in the last two decades (Branch \& Tammann 1992); however, these objects are hardly detectable at redshifts higher than $z=1.7$, so the study of more distant regions of the Universe leads to the needing to implement more powerful standard candles. The problem becomes particularly crucial at intermediate redshift, $z=6-7$, where, up to now, not very well-defined distance indicators are available.

In the last years, several efforts have been made in order to implement gamma ray bursts (GRBs), the most powerful explosions in the Universe, as standard candles, and several interesting results have recently been achieved (e.g. Amati et al. 2008; Basilakos \& Perivolaropoulos 2008, and references therein). Considering the standard model of such objects, the GRB phenomenon should originate from the black hole formation and reach huge amounts of energy (up to $10^{54} \mathrm{erg}$ ). These events are observed at considerable distances, so there are several efforts to frame them into the standard of cosmological distance ladder.

In the literature, several more-detailed models give account for the GRB formation, e.g. Meszaros (2006); Ruffini et al. (2008), but, up to now, none of them is intrinsically capable of connecting all the observable quantities. For this reason, GRBs cannot be used as standard candles. Despite this shortcoming, there are several observational correlations among the photometric and spectral properties of GRBs. These features allow use of GRBs as distance indicators (Schaefer 2007), even when they cannot be fully "enrolled" in the class of standard candles. In particular, it is possible to connect the peak energy of GRBs, $E_{\mathrm{p}}$, with the isotropic energy released in the burst, $E_{\mathrm{iso}}$, and with the rest frame jet break-time of the afterglow optical light curve, measured in days, $t_{\mathrm{b}}$, (Liang \& Zhang 2005):

$\log E_{\text {iso }}=a+b_{1} \log \frac{E_{\mathrm{p}}(1+z)}{300 \mathrm{keV}}+b_{2} \log \frac{t_{\mathrm{b}}}{(1+z) 1 \text { day }}$

where $a$ and $b_{i}$, with $i=1,2$, are calibration constants.
Another interesting result is the relation given by Ghirlanda et al. (2004). It connects the peak energy $E_{\mathrm{p}}$ with the collimationcorrected energy, or the energy release of a GRB jet, $E_{\gamma}$, where

$E_{\gamma}=\left(1-\cos \theta_{\text {jet }}\right) E_{\text {iso }}$,

with $\theta_{\text {jet }}$ the jet opening angle, given by (Sari et al. 1999):

$\theta_{\text {jet }}=0.163\left(\frac{t_{\mathrm{b}}}{1+z}\right)^{3 / 8}\left(\frac{n_{0} \eta_{\gamma}}{E_{\mathrm{iso}, 52}}\right)^{1 / 8}$,

where $E_{\text {iso }, 52}=E_{\text {iso }} / 10^{52} \mathrm{erg}, n_{0}$ is the circumburst particle density in $1 \mathrm{~cm}^{-3}$, and $\eta_{\gamma}$ the radiative efficiency. The Ghirlanda et al. relation is

$\log E_{\gamma}=a+b \log \frac{E_{\mathrm{p}}}{300 \mathrm{keV}}$,

where $a$ and $b$ are two calibration constants.

These two relations are used the most in constraining cosmology due to their relatively small scatter, interestingly very tight in the Ghirlanda et al. one, and the sufficient number of data points available.

In Schaefer (2007), an example of the discrepancy between data and theoretical curves is shown for these two relations. It is worth noticing that the calibration of the above relations is necessary to avoid the circularity problem: all the relations need to be calibrated for every set of cosmological parameters. Indeed, all GRB distances, obtained only in a photometric way, are strictly dependent on the cosmological parameters since there is no low-redshift set of GRBs to achieve a cosmologyindependent calibration.

Recently, Liang et al. (2008) present a calibration method (Liang thereafter) for several GRB relations, included the above relations (1) and (4), in a cosmology-independent way using the SNeIa. In fact, the SNeIa are very accurate standard candles, but their range is limited up to $z \approx 1.7$; hence, assuming that relations (1) and (4) work at any $z$ and that, at the same redshift, 
Table 1. Parameter values obtained by Liang et al. (2008).

\begin{tabular}{ccc}
\hline \hline Relation & $a$ & $b$ \\
\hline$E_{\gamma}-E_{\mathrm{p}}$ & $52.26 \pm 0.09$ & $1.69 \pm 0.11$ \\
$E_{\text {iso }}-E_{\mathrm{p}}-t_{\mathrm{b}}$ & $52.83 \pm 0.10$ & $2.28 \pm 0.30$ \\
& & $-1.07 \pm 0.21$ \\
\hline
\end{tabular}

GRBs and SNeIa have the same luminosity distance, it becomes possible, in principle, to calibrate GRB relations at low redshifts. The calibration parameters are shown in Table 1 . For the $E_{\text {iso }}-$ $E_{\mathrm{p}}-t_{\mathrm{b}}$ relation, the $b$-values in the first line is $b_{1}$ and in the second line is $b_{2}$.

When our working-relations are calibrated with the Liang method, we can compute the luminosity distance $d_{1}$ from the well-known relation between $d_{1}$ and the energy-flux ratio of the distance indicators in consideration. Afterwards, we can use a formulation given by Visser (2004), where the luminosity distance $d_{1}$ is related to the cosmographic parameters (Weinberg 1972) by means of a Taylor series expansion for the same $d_{1}$. Such an analysis works very well at low and intermediate redshifts, since very good classes of standard candles are available there. Besides, it is useful to constrain alternative theories of gravity, as shown in Capozziello et al. (2008). Since we are calibrating GRBs by SNeIa (in the SNeIa redshift range, the $d_{1}$ Taylor series analysis works very well), the method could also be extended to the next step (intermediate-high redshifts) where GRBs are expected to be suitable distance indicators. This working hypothesis could be useful in order to link low and high redshift ranges and then fully probe $d_{1}$. However, it is clear that such a Taylor expansion, derived for low redshifts, can be problematic for fitting GRBs at high redshifts. Here, we consider it a viable methodological approach to link GRBs to SNeIa.

The aim of this work is to achieve the cosmographic parameters (Weinberg 1972) using the above GRB relations and then to test the cosmological density parameters in a $\Lambda$ CDM model. The only assumption that we make here is that the Universe is described by a Friedmann-Robertson-Walker geometry and the scale factor of the universe $a(t)$ can be expanded in a Taylor series (Sect. 2). In Sect. 3, after considering a sample of 27 GRBs, we use a best-fit analysis to derive the cosmographic parameters discussed in the previous section, adopting the so-called Chevallier, Polarsky, Linder parameterization for the equation of state (EoS). Discussion and conclusions are given in Sect. 4.

\section{Cosmography}

The calibration we want to achieve should be cosmologically model-independent; hence, applying the above relations to a GRB sample in a given $z$-range, we want to derive the related cosmography. In particular, we want to obtain deceleration, jerk, and snap parameters (Visser 2004) and compare them with the current values deduced by other methods and observations (see, for example, Basilakos \& Perivolaropoulos 2008; Capozziello et al. 2008, and references therein).

Being only related to the derivatives of the scale factor allows to fit the cosmographic parameters versus the distance-redshift relation without any a priori assumption on the underlying cosmological model but, this fails at very high redshifts where the Taylor expansion does not work yet.
To build a distance-redshift diagram, one has to calculate the luminosity distance for each GRB in a given sample. In our case the luminosity distance is

$d_{1}=\left(\frac{E_{\text {iso }}}{4 \pi S_{\text {bolo }}^{\prime}}\right)^{\frac{1}{2}}$,

where $S_{\text {bolo }}^{\prime}=S_{\text {bolo }} /(1+z)$ is the bolometric fluence of gamma rays in the burst, corrected with respect to the rest frame. The definition of $E_{\text {iso }}$ is different for each relation used, therefore for the luminosity distance, we have

$d_{1}=\left[\frac{10^{a}\left(\frac{E_{\mathrm{p}}(1+z)}{300 \mathrm{keV}}\right)^{b_{1}}\left(\frac{t_{\mathrm{b}}}{(1+z) 1 \text { day }}\right)^{b_{2}}}{4 \pi S_{\text {bolo }}^{\prime}}\right]^{1 / 2}$,

adopting the Liang-Zhang relation, with $a, b_{1}$, and $b_{2}$ given in Table 1, and

$d_{1}=7.575 \frac{(1+z) a^{2 / 3}\left[E_{\mathrm{p}}(1+z) / 100 \mathrm{keV}\right]^{2 b / 3}}{\left(S_{\text {bolo }} t_{\mathrm{b}}\right)^{1 / 2}\left(n_{0} \eta_{\gamma}\right)^{1 / 6}} \mathrm{Mpc}$

for the Ghirlanda et al. relation, with $a$ and $b$ given in 1 (Xu et al. 2005). Note that the former gives $d_{1}$ in centimeters, therefore it divides the result for the value of 1 parsec in $\mathrm{cm}$, while the latter gives $d_{1}$ directly in Mpc.

The luminosity distance can be connected to the Hubble series (Weinberg 1972). Expanding the Hubble law up to the fourth order in redshift and considering the related luminosity distance, we get (Visser 2004)

$$
\begin{aligned}
d_{1}(z)= & d_{\mathrm{H} z}\left\{1+\frac{1}{2}\left[1-q_{0}\right] z-\frac{1}{6}\left[1-q_{0}-3 q_{0}^{2}+j_{0}+\frac{k d_{\mathrm{H}}^{2}}{a_{0}^{2}}\right] z^{2}\right. \\
& +\frac{1}{24}\left[2-2 q_{0}-15 q_{0}^{2}-15 q_{0}^{3}+5 j_{0}\left(1+2 q_{0}\right)+s_{0}\right. \\
& \left.\left.+\frac{2 k d_{\mathrm{H}}^{2}\left(1+3 q_{0}\right)}{a_{0}^{2}}\right] z^{3}+O\left(z^{4}\right)\right\}
\end{aligned}
$$

where $d_{\mathrm{H}}=c / H_{0}$ is the Hubble radius and where the cosmographic parameters are defined as

$$
\begin{aligned}
& H(t)=+\frac{1}{a} \frac{\mathrm{d} a}{\mathrm{~d} t}, \\
& q(t)=-\frac{1}{a} \frac{\mathrm{d}^{2} a}{\mathrm{~d} t^{2}}\left[\frac{1}{a} \frac{\mathrm{d} a}{\mathrm{~d} t}\right]^{-2}, \\
& j(t)=+\frac{1}{a} \frac{\mathrm{d}^{3} a}{\mathrm{~d} t^{3}}\left[\frac{1}{a} \frac{\mathrm{d} a}{\mathrm{~d} t}\right]^{-3}, \\
& s(t)=+\frac{1}{a} \frac{\mathrm{d}^{4} a}{\mathrm{~d} t^{4}}\left[\frac{1}{a} \frac{\mathrm{d} a}{\mathrm{~d} t}\right]^{-4} .
\end{aligned}
$$

They are usually referred to as the Hubble, deceleration, jerk, and snap parameters, respectively. Their present values, which we denote with a subscript 0 , may be used to characterize the evolutionary status of the Universe. For instance, $q_{0}<0$ denotes an accelerated expansion, while $j_{0}$ allows us to distinguish among different accelerating models; a positive value of $j_{0}$ indicates that, in the past, the acceleration reversed its sign. In this paper, according to the WMAP observations, we assume the value of the Hubble constant $H_{0} \simeq 70 \pm 2 \mathrm{~km} \mathrm{~s}^{-1} \mathrm{Mpc}^{-1}$ (Komatsu et al. 2008). 
Table 2. GRBs data sample.

\begin{tabular}{ccccccc}
\hline \hline $\begin{array}{c}\text { GRB } \\
(1)\end{array}$ & $\begin{array}{c}z \\
(2)\end{array}$ & $\begin{array}{c}E_{\mathrm{p}}(\mathrm{keV}) \\
(3)\end{array}$ & $\begin{array}{c}S_{\text {bolo }}(\mathrm{erg} \mathrm{cm}-2) \\
(4)\end{array}$ & $\begin{array}{c}t_{\text {jet }}(\text { days }) \\
(5)\end{array}$ & $\begin{array}{c}\theta_{\text {jet }}(\mathrm{deg} .) \\
(6)\end{array}$ & $\begin{array}{c}n_{0}\left(\mathrm{~cm}^{-3}\right) \\
(7)\end{array}$ \\
\hline 970508 & 0.84 & $389 \pm 40$ & $8.09 \mathrm{E}-6 \pm 8.1 \mathrm{E}-7$ & $25 \pm 5$ & $23 \pm 3$ & $3.0 \pm 2.4$ \\
970828 & 0.96 & $298 \pm 30$ & $1.23 \mathrm{E}-4 \pm 1.2 \mathrm{E}-5$ & $2.2 \pm 0.4$ & $5.91 \pm 0.79$ & $3.0 \pm 2.4$ \\
980703 & 0.97 & $254 \pm 25$ & $2.83 \mathrm{E}-5 \pm 2.9 \mathrm{E}-6$ & $3.4 \pm 0.5$ & $11.02 \pm 0.8$ & $28.0 \pm 10$ \\
990123 & 1.61 & $604 \pm 60$ & $3.11 \mathrm{E}-4 \pm 3.1 \mathrm{E}-5$ & $2.04 \pm 0.46$ & $3.98 \pm 0.57$ & $3.0 \pm 2.4$ \\
990510 & 1.62 & $126 \pm 10$ & $2.85 \mathrm{E}-5 \pm 2.9 \mathrm{E}-6$ & $1.6 \pm 0.2$ & $3.74 \pm 0.28$ & $0.29 \pm 0.14$ \\
990705 & 0.84 & $189 \pm 15$ & $1.34 \mathrm{E}-4 \pm 1.5 \mathrm{E}-5$ & $1 \pm 0.2$ & $4.78 \pm 0.66$ & $3.0 \pm 2.4$ \\
990712 & 0.43 & $65 \pm 10$ & $1.19 \mathrm{E}-5 \pm 6.2 \mathrm{E}-7$ & $1.6 \pm 0.2$ & $9.47 \pm 1.2$ & $3.0 \pm 2.4$ \\
991216 & 1.02 & $318 \pm 30$ & $2.48 \mathrm{E}-4 \pm 2.5 \mathrm{E}-5$ & $1.2 \pm 0.4$ & $4.44 \pm 0.7$ & $4.7 \pm 2.8$ \\
010222 & 1.48 & $309 \pm 12$ & $2.45 \mathrm{E}-4 \pm 9.1 \mathrm{E}-6$ & $0.93 \pm 0.1$ & $3.03 \pm 0.14$ & $3.0 \pm 2.4$ \\
011211 & 2.14 & $59 \pm 8$ & $9.20 \mathrm{E}-6 \pm 9.5 \mathrm{E}-7$ & $1.56 \pm 0.16$ & $5.38 \pm 0.66$ & $3.0 \pm 2.4$ \\
020124 & 3.20 & $87 \pm 18$ & $1.14 \mathrm{E}-5 \pm 1.1 \mathrm{E}-6$ & $3 \pm 0.4$ & $5.07 \pm 0.64$ & $3.0 \pm 2.4$ \\
020405 & 0.70 & $364 \pm 90$ & $1.10 \mathrm{E}-4 \pm 2.1 \mathrm{E}-6$ & $1.67 \pm 0.52$ & $6.27 \pm 1.03$ & $3.0 \pm 2.4$ \\
020813 & 1.25 & $142 \pm 14$ & $1.59 \mathrm{E}-4 \pm 2.9 \mathrm{E}-6$ & $0.43 \pm 0.06$ & $2.8 \pm 0.36$ & $3.0 \pm 2.4$ \\
021004 & 2.32 & $80 \pm 53$ & $3.61 \mathrm{E}-6 \pm 8.6 \mathrm{E}-7$ & $4.74 \pm 0.5$ & $8.47 \pm 1.06$ & $30.0 \pm 27.0$ \\
030226 & 1.98 & $97 \pm 27$ & $8.33 \mathrm{E}-6 \pm 9.8 \mathrm{E}-7$ & $1.04 \pm 0.12$ & $4.71 \pm 0.58$ & $3.0 \pm 2.4$ \\
030328 & 1.52 & $126 \pm 14$ & $6.14 \mathrm{E}-5 \pm 2.4 \mathrm{E}-6$ & $0.8 \pm 0.1$ & $3.58 \pm 0.45$ & $3.0 \pm 2.4$ \\
030329 & 0.17 & $67.9 \pm 2.3$ & $2.31 \mathrm{E}-4 \pm 2.0 \mathrm{E}-6$ & $0.5 \pm 0.1$ & $5.69 \pm 0.5$ & $1.0 \pm 0.11$ \\
030429 & 2.66 & $35 \pm 12$ & $1.13 \mathrm{E}-6 \pm 1.9 \mathrm{E}-7$ & $1.77 \pm 1.0$ & $6.3 \pm 1.52$ & $3.0 \pm 2.4$ \\
041006 & 0.71 & $63 \pm 12$ & $1.75 \mathrm{E}-5 \pm 1.8 \mathrm{E}-6$ & $0.16 \pm 0.04$ & $2.79 \pm 0.41$ & $3.0 \pm 2.4$ \\
050318 & 1.44 & $47 \pm 15$ & $3.46 \mathrm{E}-6 \pm 3.5 \mathrm{E}-7$ & $0.21 \pm 0.07$ & $3.65 \pm 0.5$ & $3.0 \pm 2.4$ \\
050505 & 4.27 & $70 \pm 23$ & $6.20 \mathrm{E}-6 \pm 8.5 \mathrm{E}-7$ & $0.21 \pm 0.04$ & $3.0 \pm 0.8$ & $3.0 \pm 2.4$ \\
050525 & 0.61 & $81.2 \pm 1.4$ & $2.59 \mathrm{E}-5 \pm 1.3 \mathrm{E}-6$ & $0.28 \pm 0.12$ & $4.04 \pm 0.8$ & $3.0 \pm 2.4$ \\
050904 & 6.29 & $436 \pm 200$ & $2.0 \mathrm{E}-5 \pm 2 \mathrm{E}-6$ & $2.6 \pm 1$ & $8 \pm 1$ & $3.0 \pm 2.4$ \\
051022 & 0.80 & $510 \pm 22$ & $3.40 \mathrm{E}-4 \pm 1.2 \mathrm{E}-5$ & $2.9 \pm 0.2$ & $4.4 \pm 0.1$ & $3.0 \pm 2.4$ \\
060124 & 2.30 & $237 \pm 76$ & $3.37 \mathrm{E}-5 \pm 3.4 \mathrm{E}-6$ & $1.2 \pm$ & $3.72 \pm 0.15$ & $3.0 \pm 2.4$ \\
060210 & 3.91 & $149 \pm 35$ & $1.94 \mathrm{E}-5 \pm 1.2 \mathrm{E}-6$ & $0.33 \pm 0.08$ & $1.9 \pm 0.17$ & $3.0 \pm 2.4$ \\
060526 & 3.21 & $25 \pm 5$ & $1.17 \mathrm{E}-6 \pm 1.7 \mathrm{E}-7$ & $1.27 \pm 0.35$ & $4.7 \pm 1$ & $3.0 \pm 2.4$ \\
\hline
\end{tabular}

References: (1) Jimenez et al. (2001); (2) Metzger et al. (1997); (3) Djorgovski et al. (1999); (4) Kulkarni et al. (1999); (5) Israel et al. (1999); (6) Bjornsson et al. (2001); (7) Li et al. (2008)

The cosmographic parameters can be expressed in terms of the dark energy density and the EoS. Following the prescriptions of the Dark Energy Task Force, (Albrecht et al. 2006), we use the Chevallier-Polarski-Linder parameterization (CPL) for the EoS (Chevallier et al. 2001; Linder 2003) and assume a spatially flat Universe filled with dust matter and dark energy. The dimensionless Hubble parameter $E(z)=H / H_{0}$ reads as

$E^{2}(z)=\Omega_{\mathrm{M}}(1+z)^{3}+\Omega_{\mathrm{X}}(1+z)^{3\left(1+w_{0}+w_{a}\right)} \mathrm{e}^{-\frac{3 w_{a} z}{1+z}}$,

with $\Omega_{\mathrm{X}}=1-\Omega_{\mathrm{M}}$ and $w_{0}$ and $w_{a}$ the CPL parameterization for the EoS (see Chevallier et al. 2001; Linder 2003; Capozziello et al. 2008, for details). We can have $\Omega_{X} \equiv \Omega_{\Lambda}$, with $\Lambda$ the cosmological constant. Such a relation can be used to evaluate the cosmographic parameters, obtaining

$$
\begin{aligned}
q_{0}= & \frac{1}{2}+\frac{3}{2}\left(1-\Omega_{\mathrm{M}}\right) w_{0}, \\
j_{0}= & 1+\frac{3}{2}\left(1-\Omega_{\mathrm{M}}\right)\left[3 w_{0}\left(1+w_{0}\right)+w_{a}\right], \\
s_{0}= & -\frac{7}{2}-\frac{33}{4}\left(1-\Omega_{\mathrm{M}}\right) w_{a} \\
& -\frac{9}{4}\left(1-\Omega_{\mathrm{M}}\right)\left[9+\left(7-\Omega_{\mathrm{M}}\right) w_{a}\right] w_{0} \\
& -\frac{9}{4}\left(1-\Omega_{\mathrm{M}}\right)\left(16-3 \Omega_{\mathrm{M}}\right) w_{0}^{2} \\
& -\frac{27}{4}\left(1-\Omega_{\mathrm{M}}\right)\left(3-\Omega_{\mathrm{M}}\right) w_{0}^{3} .
\end{aligned}
$$

For a $\Lambda$ CDM-universe, where $\left(w_{0}, w_{a}\right)=(-1,0)$, it becomes

$$
\begin{aligned}
& q_{0}=-1+\frac{3}{2} \Omega_{\mathrm{M}} \\
& j_{0}=1 \\
& s_{0}=1-\frac{9}{2} \Omega_{\mathrm{M}}
\end{aligned}
$$

that are the quantities which we are going to fit using a given GRB sample.

\section{GRB data fitting}

Let us take a GRB sample into account that satisfies the above relations. Unfortunately only 27 GRBs have observed jet breaks in the Schaefer sample (Schaefer 2007). The observational quantities of GRBs to take into account, are listed in Table 2. The luminosity distance for each of the relations is given by Eqs. (6) and (7), and then we obtain a data distribution in the luminosity distance-redshift diagram $d_{1}-z$. The errors on the data are only of a photometric nature and, in a first analysis, we can exclude errors on the redshift. For each GRB, we assume $\eta_{\gamma}=0.2$ and $\sigma_{\eta}=0$, (Frail et al. 2001).

Another version of the Hubble series can be used to improve the data fit. If we consider the equation for the distance modulus,

$\mu=25+\frac{5}{\ln (10)} \ln \left[d_{1} /(1 \mathrm{Mpc})\right]+25$, 
Table 3. Results of the fits. LZ is for Liang-Zhang relation, GGL for the Ghirlanda et al. one.

\begin{tabular}{cccc}
\hline \hline Fit & $q_{0}$ & $j_{0}+\Omega$ & $s_{0}$ \\
\hline$d_{1}(z) \mathrm{LZ}$ & $-0.94 \pm 0.30$ & $2.71 \pm 1.1$ & \\
$d_{1}(z) \mathrm{GGL}$ & $-0.39 \pm 0.11$ & $2.52 \pm 1.33$ & \\
$\ln \left[d_{1} / z\right] \mathrm{LZ}$ & $-0.68 \pm 0.30$ & $0.021 \pm 1.07$ & $3.39 \pm 17.13$ \\
$\ln \left[d_{1} / z\right] \mathrm{GGL}$ & $-0.78 \pm 0.20$ & $0.62 \pm 0.86$ & $8.32 \pm 12.16$ \\
\hline
\end{tabular}

and substitute the equation for $d_{1}$, we obtain a logarithmic version of the Hubble series:

$$
\begin{aligned}
\ln \left[d_{1} /(z \mathrm{Mpc})\right]= & \ln \left(d_{\mathrm{H}} / \mathrm{Mpc}\right)-\frac{1}{2}\left[-1+q_{0}\right] z \\
& +\frac{1}{24}\left[-3+10 q_{0}+9 q_{0}^{2}-4\left(j_{0}+1+\frac{k d_{\mathrm{H}}^{2}}{a_{0}^{2}}\right)\right] z^{2} \\
& +\frac{1}{24}\left[4 q_{0}\left(j_{0}+1+k d_{\mathrm{H}}^{2} a_{0}^{2}\right)+5-9 q_{0}-16 q_{0}^{2}\right. \\
& \left.-10 q_{0}^{3}+j_{0}\left(7+4 q_{0}\right)+s_{0}\right] z^{3}+O\left(z^{4}\right) .
\end{aligned}
$$

This logarithmic version shows the advantage when there is no need to transform the uncertainties on the distance modulus. With these considerations in mind, we perform a polynomial least-squares fit for each relation of the data assuming Taylor series polynomials, both in distance and in logarithmic distance. We stop at order $n=3$ both for the polynomial fit and for the logarithmic fit. In the latter case, we obtain an estimate of the snap parameter. Note that we are using least squares since, in absence of any better data-fitting procedure, this is the standard procedure when assuming Gaussian distributed uncertainties.

The truncated polynomial used in the fits has the form

$d(z)=\sum_{i=1}^{3} a_{i} z^{i}$,

and

$$
\ln [d(z) /(z \mathrm{Mpc})]=\sum_{i=1}^{3} b_{i} z^{i}
$$

for the logarithmic fit. In the latter case, the Hubble constant enters as the $i=1$ component of the fit. As stated above, we use $H_{0}$ as a constraint (a prior).

The fits can be used to estimate the deceleration and the jerk parameters. The logarithmic fit is better for estimating the snap parameter through the values of the coefficients $a_{i}$ and $b_{i}$ and their statistical uncertainties. The statistical uncertainties on $q_{0}$ are linearly related to the statistical uncertainties on the parameter $b_{1}$, while the statistical uncertainties on $j_{0}$ and $s_{0}$ depend non-linearly on $q_{0}$ and its statistical uncertainty. It is worth noticing the combination $j_{0}+k d_{\mathrm{H}}^{2} / a_{0}^{2}$, which is a well-known degeneracy in Eq. (21) (Weinberg 1972). It means that we cannot determine $j_{0}$ and $\Omega=1+k d_{\mathrm{H}}^{2} / a_{0}^{2}$ separately, but we need an independent determination of $\Omega$ to estimate the value of the jerk parameter.

The results of the fits are presented in Table 3, and all of them include the error on the data. For the calculation of the uncertainties on $d_{1}$, we have followed the procedure discussed in $\mathrm{Xu}$ et al. (2005). For example, the fractional uncertainties on $d_{1}$ in the Ghirlanda et al. relation,without the small angle
Table 4. Goodness of the fits with the R-square.

\begin{tabular}{cc}
\hline \hline Fit & R-square \\
\hline$d_{1}(z) \mathrm{LZ}$ & 0.9909 \\
$d_{1}(z) \mathrm{GGL}$ & 0.9977 \\
$\ln \left[d_{1} / z \mathrm{Mpc}\right] \mathrm{LZ}$ & 0.4005 \\
$\ln \left[d_{1} / z \mathrm{Mpc}\right] \mathrm{GGL}$ & 0.2929 \\
\hline
\end{tabular}

approximation for $\theta_{\text {jet }}$ (Sari et al. 1999), are given by

$$
\begin{aligned}
\left(\frac{\sigma_{d_{1}}}{d_{1}}\right)^{2}= & \frac{1}{4}\left[\left(\frac{\sigma_{S_{\text {bolo }}}}{S_{\text {bolo }}}\right)^{2}\right]+\frac{1}{4} \frac{1}{\left(1-\sqrt{C_{\theta}}\right)^{2}}\left[\left(\frac{\sigma_{a}}{a}\right)^{2}+\left(b \frac{\sigma_{E_{\mathrm{p}}}}{E_{\mathrm{p}}}\right)^{2}\right. \\
& \left.+\left(b \frac{\sigma_{\mathrm{b}}}{b} \ln \frac{E_{\mathrm{p}}}{100}\right)^{2}\right]+\frac{1}{4} \frac{C_{\theta}}{\left(1-\sqrt{C_{\theta}}\right)^{2}} \\
& \times\left[\left(\frac{3 \sigma_{t_{\mathrm{b}}}}{t_{\mathrm{b}}}\right)^{2}+\left(\frac{\sigma_{n_{0}}}{n_{0}}\right)^{2}\right],
\end{aligned}
$$

where $C_{\theta}=[\theta \sin \theta / 8-8 \cos \theta]^{2}$. This shows that the uncertainties on $d_{1}$ in the Ghirlanda et al. relation are very high, due to the dependence on several parameters. For this reason, in Fig. 1, the prediction bounds are plotted at a $68 \%$ confidence level, instead of $95 \%$, as in LZ-relation.

As said above, only statistical uncertainties have been considered and other kinds of errors (systematics of cosmological inference, modelling errors and "historical" biases, Visser 2007b) have been neglected. If we do not assume $H_{0}$ as a constraint, the analysis gives $H_{0}=56 \mathrm{~km} \mathrm{~s}^{-1} \mathrm{Mpc}^{-1}$, which means that the data sample needs to be improved with further GRBs to give more reliable results.

Another step would be to test the goodness of the next fit statistics using the MATLAB package. In particular, we used the R-square method: a value closer to 1 indicates a better fit. In Table 4, the results of R-square are shown and the plots of the residuals of the fits are shown in Figs. 1, 2. For the logarithmic fit, the bad value of the R-square is caused by the logarithm of the Hubble series, which spreads a lot of the data on the $\ln \left(d_{1}\right)$-axis. The values $\ll 1$ for the logarithmic fits are due to the discrepancy of the data.

In summary, the results are in quite good agreement with the $\Lambda C D M$ model, giving a Universe model that accelerates in the present epoch and that has undergone a decelerated phase in the past. The signature of this past phase is related to the sign change of the parameter $q_{0}$ and the positive value of the jerk parameter, unless a positive value of the spatial curvature constant $k$ is considered. However this occurrence is excluded by the last observational results, which confirm a spatially flat Universe (Komatsu et al. 2008).

\subsection{The CPL parameterization to test the $\Lambda C D M$ model}

As said, the cosmographic parameters may also be expressed in terms of the dark energy density and EoS parameters. Starting from the Friedmann equation, we obtain the Hubble parameter:

$H^{2}=\left(\frac{8 \pi G}{3}\right) \rho$

where $\rho$ is the energy density. The continuity equation for each cosmological component is given by the Bianchi identity (Weinberg 1972):

$\frac{\dot{\rho}}{\rho}=-3 H\left(1+\frac{p}{\rho}\right)=-3 H[1+w(z)]$, 


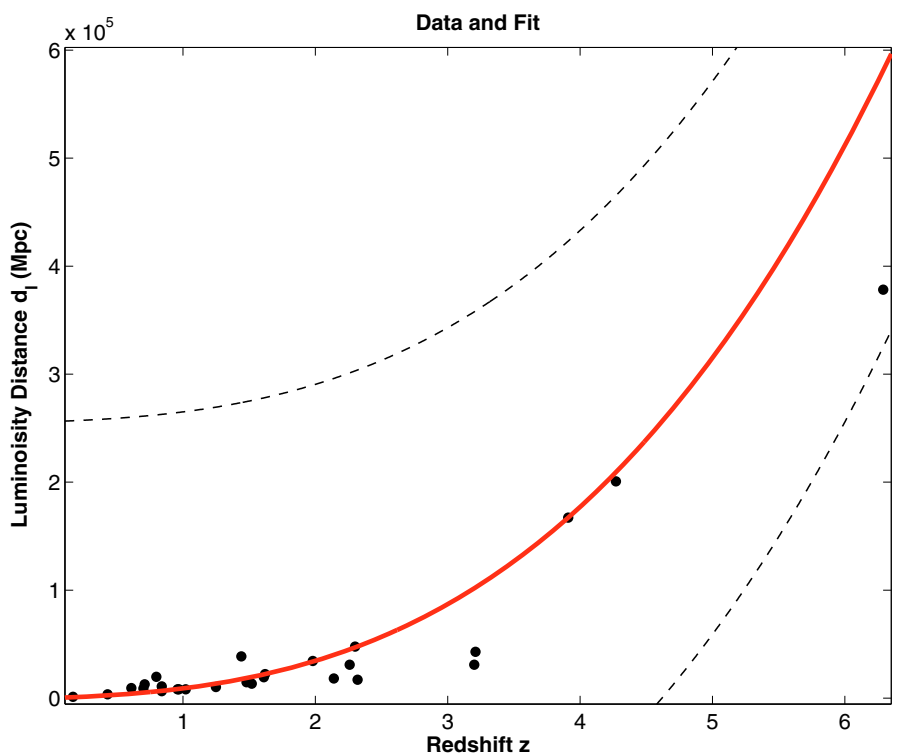

Fig. 1. Luminosity distance-redshift diagram and the residuals of the $d_{1}(z)$ GGL fit. Note the discrepancy at high- $z$. The dotted lines are the bounds predicted at $68 \%$ confidence level.

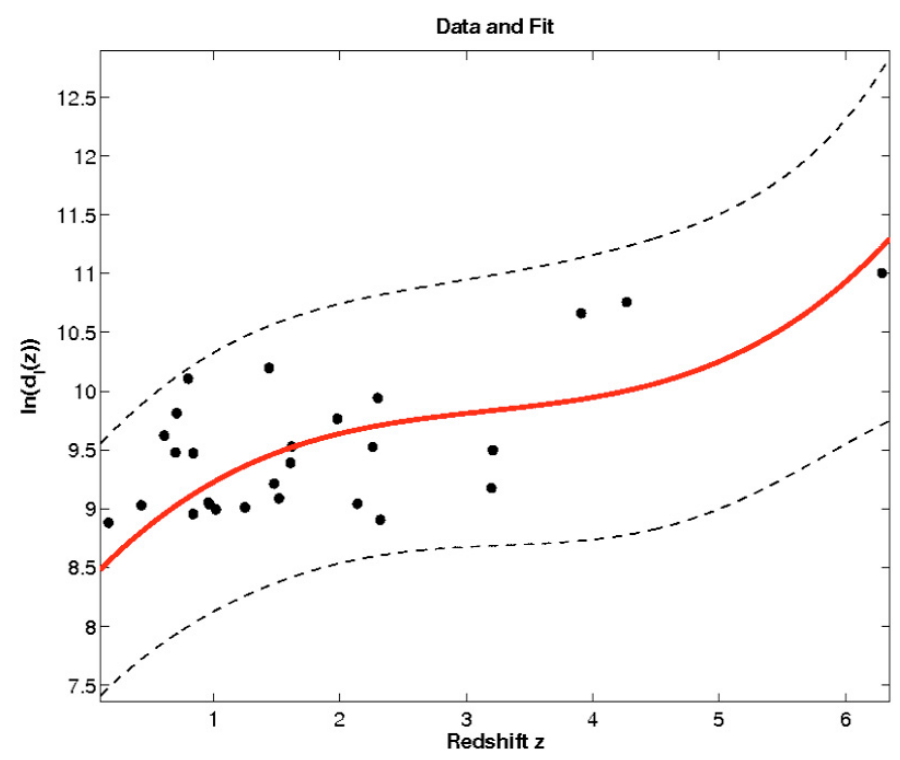

Fig. 2. Logarithmic version of the luminosity distance relation versus redshift and the residuals of the $\ln \left[d_{1} / z\right.$ Mpc $]$ LZ fit. In this version of the Hubble series, the discrepancy is the same at every $z$. The dotted lines are the bounds predicted at $95 \%$ confidence level.

where $p$ is the pressure of the component considered and $w(z)=$ $p / \rho$ the redshift-dependent EoS for each component. The dark energy component responsible for the observed acceleration of the universe must have a negative EoS, (Riess et al. 2004; Allen et al. 2004). To find $w(z)_{\mathrm{DE}}$, we can adopt the CPL parameterization, (Chevallier et al. 2001; Linder 2003), where

$w(z)_{\mathrm{DE}}=w_{0}+w_{a} z\left(\frac{1}{1+z}\right)$

with $w_{0}$ and $w_{a}$ two parameters that enter directly into the equations for the cosmographic parameters, (14-16). To test the $\Lambda \mathrm{CDM}$ model, we have assumed $\left(w_{0}, w_{a}\right)=(-1,0)$. Conversely, such a case can be generalized by deducing the value of cosmographic parameters $\left(\Omega_{\mathrm{M}}, w_{0}, w_{a}\right)$ from polynomial fits where
Table 5. Cosmological density parameters.

\begin{tabular}{ccc}
\hline \hline Fit & $\Omega_{\mathrm{M}}$ & $\Omega_{\Lambda}$ \\
\hline$d_{1}(z) \mathrm{LZ}$ & $0.04 \pm 0.03$ & $0.65 \pm 0.73$ \\
$d_{1}(z) \mathrm{GGL}$ & $0.46 \pm 0.43$ & $0.54 \pm 2.82$ \\
$\ln \left[d_{1} / z \mathrm{Mpc}\right] \mathrm{LZ}$ & $0.37 \pm 0.31$ & $0.63 \pm 1.13$ \\
$\ln \left[d_{1} / z \mathrm{Mpc}\right] \mathrm{GGL}$ & $0.28 \pm 0.30$ & $0.72 \pm 1.09$ \\
\hline
\end{tabular}

GRB data are considered. Adopting our GRB sample, we obtained the following values

$w_{0}=-0.53 \pm 0.64 \quad w_{a}=0.59 \pm 0.77$,

which directly enter into the CPL parameterization. The errors on the CPL parameters are directly connected with the errors on the cosmographic parameters, as easily seen from the system (14-16). The values of $w_{0}$ and $w_{a}$ agree, within the errors, with the $\Lambda \mathrm{CDM}$ model, without assuming constraints a priori on the cosmological model.

\section{Discussion and conclusions}

Starting from some relations connecting the observable quantities of GRBs, we have used a sample of 27 GRBs to derive the luminosity distance-redshift diagram of the Hubble law. The relations conveniently calibrated by SNeIa to make them independent of any cosmological models.

We have taken the Hubble law into account in the Taylor series form, assuming the luminosity distance $d_{1}$ as a redshift function whose coefficients are combinations of the cosmographic parameters $H_{0}, q_{0}, j_{0}$, and $s_{0}$. The aim was to evaluate such parameters starting from the GRB data. A direct analysis of the fits leads to the conclusion that, in the error range, the SNeIa results can also be extended at higher redshifts (Visser 2007b). Besides, such results agree with the $\Lambda$ CDM model according to Eqs. (17)-(19). In particular, the value of the parameter $q_{0}$ that we found is in agreement with the observed $\Omega_{\mathrm{M}}$ (see Table 5).

However, the sample we used is quite poor at high redshifts and, in some sense, this justifies the use of the method of Taylor series which works very well at low redshifts. In particular, at $z>6$, we only have one GRB, GRB050904 (see Fig. 1). This GRB is very important in the fit results because it affects the trend of the fits. For this reason we need some richer sample at medium-high redshifts to constrain the results better. However, if we had richer samples at high redshifts, the Taylor series analysis would fail to constrain cosmological models since an exact, and not approximated, $d_{1}(z)$ expression is needed in that case. The best constraint, however, would be an absolute relation between several, GRB observables which would make the GRBs a powerful standard candle at intermediate-high redshift.

Considering these preliminary results, it seems that cosmography by GRBs could be a useful tool in constraining selfconsistent cosmological models even if, up to now, GRBs are not standard candles in the proper sense.

Acknowledgements. We thank the referee for the useful suggestions that improved the paper.

\section{References}

Albrecht, A., et al., 2006 [arXiv: astro-ph/0609591]

Allen, S. W., Schmidtn R. W., \& Ebeling, H. 2004, MNRAS, 353, 457

Amati, L., et al. 2008 [arXiv:0805. 0377]

Basilakos, S., \& Perivolaropoulos, L. 2008 [arXiv:0805. 0875] 
Bjornsson, G., Hjorth, J., Jakobsson, P., Christensen, L., \& Holland, S. 2001, ApJ, 552, L121

Branch, D., \& Tammann, G. A. 1992, ARA\&A, 30, 359

Capozziello, S., et al. 2008, to appear in PRD [arXiv:0802.1583]

Chevallier, M., \& Polarski, D. 2001, Int. J. Mod. Phys. D. 10, 213

Linder, E. V. 2003, Phys. Rev. Lett., 90, 091301

Djorgovski, S. G., Kulkarni, S. R., Bloom, J. S., et al. 1999b, GCN Circ., 189, http://gcn.gsfc.nasa.gov/gcn/gcn3/189.gcn3

Frail, D. A., Kulkarni, S.R., \& Sari, R. 2001, ApJ, 562, L55

Ghirlanda, G., Ghisellini, G., \& Lazzati, D. 2004, ApJ, 616, 331

Israel, G., Marconi, G., \& Covino, S. 1999, A\&A, 348, L5

Jimenez, R., Band, D., \& Piran, T. 2001, ApJ, 561, 171

Komatsu, E., Djorgovski, S. G., \& Odewahn, S. C. 2008 [arXiv:0803.0547]

Kulkarni, S. R., Djorgovski, S. G., Odewahn, S. C., et al. 1999, Nature, 398, 389

Li, H., Xia, J.-Q., \& Liu, J. 2008, ApJ, 680, 92
Liang, E., \& Zhang, B. 2005, ApJ, 633, 611

Liang, N., et al. 2008 [arXiv: 0802 . 4262]

Meszaros, P. 2006, Rept. Prog. Phys., 69, 2259

Metzger, M. R., Djorgovski, S. G., Kulkarni, S. R., et al. 1997, Nature, 387, 878

Qi, S., Wang, F., \& Lu, T. 2008 [arXiv : 0803.4304]

Riess, A. G., Strolger, L.-G., \& Tonry, J. 2004, ApJ, 607, 665

Ruffini, R., et al. 2008, Gamma Ray Bursts, Proceedings XI Marcel Grossmann Meeting (Singapore: World Scientific)

Sari, R., Piran, T., \& Halpern, J. P. 1999, ApJ, 519, L17

Schaefer, B. E. 2007, ApJ, 660, 16

Visser, M. 2004, Class. Quant. Grav., 21, 2603

Visser, M., \& Cattoën, C. 2007a, Class. Quant. Grav., 24, 5985

Visser, M., \& Cattoën, C. 2007b [arXiv:gr-qc/0703122]

Weinberg, S. 1972, Gravitation and Cosmology: Principles and applications of the general theory of relativity (New York: Wiley)

Xu, D., Dai, Z. G., \& Liang, E. W. 2005, ApJ, 633, 603 